Pacific Journal of Mathematics

AN INDEX THEOREM AND HYPOELLIPTICITY ON 


\title{
AN INDEX THEOREM AND HYPOELLIPTICITY ON NILPOTENT LIE GROUPS
}

\author{
KenNeth G. MilleR
}

\begin{abstract}
Extending results of Grushin we determine the index of $p(x, D)$ where $p(x, \xi)$ is a polynomial homogeneous with respect to some family of dilations on $\boldsymbol{R}^{2 d}$ and $p(x, \xi) \neq 0$ if $(x, \xi) \neq(0,0)$. In general these operators are not elliptic. If $G$ is a step two nilpotent Lie group and $P$ is a left invariant differential operator on $G$ which is homogeneous with respect to some family of dilations, we apply this index theorem to prove that $P$ is hypoelliptic if and only if $P^{*}$ is hypoelliptic. This extends a result of Helffer and Nourrigat.
\end{abstract}

1. An index theorem. A family of dilations on a Lie algebra $\mathscr{G}$ is a one parameter family of automorphisms $\left\{\delta_{r}: r>0\right\}$ of $\mathscr{G}$ of the form $\delta_{r}=\exp ((\log r) A)$, where $A$ is a diagonalizable automorphism of $\mathscr{G}$ with positive real eigenvalues. There is no loss of generality in assuming that the smallest eigenvalue is 1 . A finite dimensional normed vector space $V$ with norm | | determines an abelian Lie algebra. Let $\left\{\delta_{r}\right\}$ be a family of dilations on $V$. For $w \in V$ define $\|w\|$ by $\|w\|=r$ if $\left|\delta_{r}^{-1}(w)\right|=1$. Then $w \rightarrow\|w\|$ is continuous on $V$ and $C^{\infty}$ on $V-\{0\}$ by the implicit function theorem. Let $\mathscr{B}=$ $\left\{w_{1}, w_{2}, \cdots, w_{n}\right\}$ be a basis for $V$ consisting of eigenvectors of $A$ with corresponding eigenvalues $\mu_{1}, \cdots, \mu_{n}$. If $w=a_{1} w_{1}+\cdots+a_{n} w_{n}$, then

$$
\begin{gathered}
\delta_{r} w=\sum r^{\mu_{j}} a_{j} w_{j} \quad \text { and } \\
\|w\| \approx \sum\left|a_{j}\right|^{1 / \mu_{j}} .
\end{gathered}
$$

Throughout this section we will be considering a family of dilations on the abelian Lie algebra $\boldsymbol{R}^{2 d}=\boldsymbol{R}_{x}^{d} \oplus \boldsymbol{R}_{\xi}^{d}$. We do not necessarily assume that either $\boldsymbol{R}_{x}^{d}$ or $\boldsymbol{R}_{\xi}^{d}$ is invariant under $\left\{\delta_{r}\right\}$. Let $f \in C^{\infty}\left(\boldsymbol{R}^{2 d}\right), f(w)=0$ for $\|w\| \leqq 1 / 2$, and $f(w)=1$ for $\|w\| \geqq 1$. Define $\Phi(w)=1+f(w)\|w\|$ and $\phi(w)=1$ for all $w=(x, \xi) \in \boldsymbol{R}^{2 d}$. Note that there is a $C$ such that if $\left|w-w^{\prime}\right| \leqq \Phi(w)$ then $\Phi\left(w^{\prime}\right) \leqq C \Phi(w)$. Thus $(\Phi, \varphi)$ is a pair of weight functions on $\boldsymbol{R}_{x}^{d}$ as defined in Beals [1]. We will usually not mention $\varphi$ and will refer to $\Phi$ as the weight function for the family of dilations $\left\{\delta_{r}\right\}$. Note that $\Phi$ satisfies the coercive estimate

$$
|w| \leqq C \Phi(w)^{\bar{\mu}}
$$

where $\bar{\mu}=\max \left\{\mu_{1}, \cdots, \mu_{2 d}\right\}$. 
For $m \in \boldsymbol{R}$, let $S_{\psi}^{m}$ denote the set of all smooth functions $p$ on $\boldsymbol{R}^{2 d}$ such that for each $\alpha$ and $\beta \in \boldsymbol{N}^{d}$

$$
\sup \left\{\Phi(x, \xi)^{-m+|\alpha|}\left|D_{\xi}^{\alpha} D_{x}^{\beta} p(x, \xi)\right|:(x, \xi) \in R^{2 d}\right\}<\infty .
$$

$\mathscr{L}_{\psi}^{m}$ is the set of pseudodifferential operators with symbols in $S_{\nu}^{m}$, $H_{\phi}^{m}$ is the associated (global) Sobolev space as defined in [1] and \|\|$_{m, \phi}$ is a norm for the topology on $H_{\phi}^{m}$. We note that in the special case where $m \in N$ and $m / \mu_{j} \in N$ for all $j$ (this is necessarily the case in the context of Theorem 2 below, by Proposition 1.3 of [7]), then \|\|$_{m, n}$ can be given explicitly as follows: Let $\mathscr{B}$ be a basis for $\boldsymbol{R}^{2 d}$ consisting of eigenvectors for $\left\{\delta_{r}\right\}$ and let $a_{j}(x, \xi)$ be the $j$ th coordinate of $(x, \xi)$ with respect to the basis $\mathscr{B}$. By (1.2) above and 6.17 of [1]

$$
\|u\|_{m, \downarrow} \approx \sum\left\|a_{j}(x, D)^{m / \mu_{j}} u\right\|+\|u\|
$$

where ||$\|$ is the $L^{2}$ norm.

We shall denote by $\widetilde{S}_{\phi}^{m}$ the subset of $S_{\nu}^{m}$ consisting of functions $p$ such that for all $\alpha$ and $\beta$ in $N^{d}$

$$
\sup \left\{\Phi(x, \xi)^{-m+|\alpha|+|\beta|}\left|D_{\xi}^{\alpha} D_{x}^{\beta} p(x, \xi)\right|:(x, \xi) \in \boldsymbol{R}^{2 d}\right\}<\infty .
$$

We say that $p \in C^{\infty}\left(\boldsymbol{R}^{2 d}\right)$ is homogeneous of degree $m$ with respect to $\left\{\delta_{r}\right\}$ for large $w$ if there is a $c, 0<c<1$, such that $p\left(\delta_{r} w\right)=r^{m} p(w)$ for all $r \geqq 1$ and all $w$ for which $\|w\| \geqq c$. If $p$ is homogeneous of degree $m$ with respect to $\left\{\delta_{r}\right\}$ for large $w$ and if $v$ is an eigenvector for the generator $A$ of $\left\{\delta_{r}\right\}$ with eigenvalue $\mu$, then

$$
r^{\mu} D_{v} p\left(\delta_{r} w\right)=r^{m} D_{v} p(w) \text {. }
$$

If $\|w\| \geqq 1$, let $r=\|w\|$ and $w^{\prime}=\delta_{r}^{-1}(w)$. Then $\left\|w^{\prime}\right\|=1$ and $D_{v} p(w)=\|w\|^{m-\mu} D_{v} p\left(w^{\prime}\right)$. Thus there is a $C$ such that

$$
\left|D_{v} p(w)\right| \leqq C\|w\|^{m-\mu} \leqq C\|w\|^{m-1}
$$

for all $w,\|w\| \geqq 1$. Consequently if $p$ is homogeneous of degree $m$ with respect to $\left\{\delta_{r}\right\}$ for large $w$, then $p \in \widetilde{S}_{\psi}^{m}$. It follows from this remark that $\Phi \in \widetilde{S}_{\Phi}^{1}$ and hence $\Phi^{m} \in \widetilde{S}_{\Phi}^{m}$ for all $m \in \boldsymbol{R}$.

We say that $p \in S_{\Phi}^{m}$ is $\Phi$-elliptic if there is a $C$ such that $\Phi(w)^{m} \leqq$ $C|p(w)|$ for $|w| \geqq C$. Note that if $p$ is a polynomial and $p$ is homogeneous of degree $m$ with respect to $\left\{\delta_{r}\right\}$, then $p$ is $\Phi$-elliptic if and only if $p(w) \neq 0$ for $|w| \neq 0$. Note that in general $\Phi$-ellipticity does not imply ellipticity in the usual sense. For example on $\boldsymbol{R}^{2} \times \boldsymbol{R}^{2}$, $p(x, \xi)=\xi_{1}^{4}+x_{1}^{2}+2 x_{1} \xi_{1}+\xi_{1}^{2}+\xi_{2}^{2}+x_{2}^{2}$ is $\Phi$-elliptic and homogeneous of degree two, where the dilations are given in terms of coordinates $a_{1}=\xi_{1}, a_{2}=x_{1}+\xi_{1}, a_{3}=\xi_{2}$ and $a_{4}=x_{2}$, with $\mu_{1}=2, \mu_{2}=\mu_{3}=\mu_{4}=1$. If $\Gamma$ is an oriented curve and $p$ maps the range of $\Gamma$ into 
$C-\{0\}$, let $\Delta_{\Gamma}$ arg $p$ denote the change in the argument of $p$ along $\Gamma$. In the following theorem $\Gamma$ is the curve in $\boldsymbol{R}_{x} \oplus \boldsymbol{R}_{\xi}$ given by $x(\theta)=\cos \theta, y(\theta)=\sin \theta, 0 \leqq \theta \leqq 2 \pi$. In the case where $\boldsymbol{R}_{\xi}^{d}$ and $\boldsymbol{R}_{x}^{d}$ are eigenspaces for $A$ with eigenvalues 1 and $1+\delta$ respectively, $\delta>0$, this theorem was proved in [2].

THEOREM 1. Let $\delta_{r}=\exp ((\log r) A), r>0$, be a family of dilations on $\boldsymbol{R}^{2 d}, \Phi$ the weight function for $\left\{\delta_{r}\right\}$. Let $p=p_{0}+p_{1}$ where $p_{0}$ is $\Phi$-elliptic and homogeneous of degree $m$ with respect to $\left\{\delta_{r}\right\}$ for large $w$ and $p_{1} \in S_{\Phi}^{m_{1}}$ for some $m_{1}<m$. Then $p(x, D): H_{\phi}^{m} \rightarrow L^{2}$ is Fredholm. If $d>1$, then ind $p(x, D)=0$. If $d=1$, then $2 \pi$ ind $p(x, D)=\Delta_{\Gamma}$ arg $p_{0}$. If $d=1$ and $p_{0}$ is a polynomial, then ind $p(x, D)$ is also given by (1.6) below.

Proof. By Theorem 7.2 of [1] and (1.3) above, $p(x, D): H_{\Phi}^{m} \rightarrow L^{2}$ is Fredholm. By Corollary 6.13 of $[1], p_{1}(x, D): H_{\phi}^{m} \rightarrow L^{2}$ is compact. Hence ind $p_{0}(x, D)=$ ind $p(x, D)$. Let $f \in C^{\infty}\left(\boldsymbol{R}^{2 d}\right)$ be real valued, $f(w)=0$ for $\|w\| \leqq 1 / 2, \quad f(w)=1$ for $\|w\| \geqq 1$. Let $a(w)=$ $f(w) /\|w\|^{m / 2}, q=p_{0} a^{2}$. Then $A=a(x, D) \in \mathscr{L}_{\Phi}^{-m / 2}$, and by the pseudodifferential operator calculus $p_{0}(x, D) A^{*} A=q(x, D)+R$ where $R \in \mathscr{L}_{\Phi}^{-1}$. Thus ind $q(x, D)=$ ind $p_{0}(x, D)$. Also $q\left(\delta_{r} w\right)=p_{0}(w) \neq 0$ for all $r \geqq 1$ and all $w,\|w\|=1$. If $d>1,\left\{w \in R^{2 d}:\|w\|=1\right\}$ is simply connected, so $q$ can be continuously deformed to a nonzero constant through $\Phi$-elliptic symbols which are homogeneous of degree 0 for large $w$. Hence ind $q(x, D)=0$.

Now consider the case $d=1$. Although $q$ is not elliptic in the classic sense, $q$ is included in the class of symbols for which Hormander proves the index theorem in $\S 7$ of [5]. In [5] it is shown that $2 \pi$ ind $q^{w}(x, D)=\Delta_{\Gamma}$ arg $q$, where $q^{w}(x, D)$ is the Weyl pseudodifferential operator with symbol $q$. By (4.10) of [5] $q^{w}(x, D)=$ $a(x, D)$ where $a=q+r, r \in S_{\Phi}^{-1}$. Thus ind $q(x, D)=$ ind $q^{w}(x, D)$. Clearly $\Delta_{\Gamma}$ arg $q=\Delta_{\Gamma} \arg p_{0}$.

If $d=1$ and $p_{0}$ is a polynomial, then ind $p(x, D)$ can also be computed as follows: Let $v_{1}$ and $v_{2}$ be eigenvectors for the generator $A$ of $\left\{\delta_{r}\right\}$, chosen so that if $\left(x_{1}, \xi_{1}\right)$ and $\left(x_{2}, \xi_{2}\right)$ are the respective $x, \xi$ coordinates of $v_{1}$ and $v_{2}$, then $x_{1} \xi_{2}-x_{2} \xi_{1}>0$. Let $\Gamma_{+}$be the

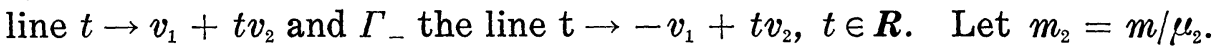
Let $\nu_{+}$be the number of complex roots $z$ of $p_{0}\left(v_{1}+z v_{2}\right)$ with positive imaginary part and $\nu_{-}$the number of complex roots of $p_{0}\left(-v_{1}+z v_{2}\right)$ with negative imaginary part. By the homogeneity of $p_{0}$,

$$
\begin{aligned}
& \Delta_{\Gamma} \arg p_{0}=\Delta_{\Gamma_{+}} \arg p_{0}-\Delta_{\Gamma_{-}} \arg p_{0} \quad \text { and } \\
& \quad \Delta_{\Gamma_{+}} \arg p_{0}=-i \int_{-\infty}^{\infty} \frac{d}{d t}\left|p_{0}\left(v_{1}+t v_{2}\right)\right| d t=2 \pi\left(\nu_{+}-m_{2} / 2\right) .
\end{aligned}
$$




$$
\Delta_{\Gamma_{-}} \arg p_{0}=-i \int_{-\infty}^{\infty} \frac{d}{d t}\left|p_{0}\left(t v_{2}-v_{1}\right)\right| d t=2 \pi\left(m_{2} / 2-\nu_{-}\right) .
$$

Thus

$$
\text { ind } p(x, D)=\nu_{+}+\nu_{-}-m_{2} \text {. }
$$

2. Hypoellipticity of $P^{*}$. Let $\mathscr{G}$ be a nilpotent Lie algebra of step 2; i.e., $\left[\mathscr{G}, \mathscr{G}_{2}\right]=0$ where $\mathscr{G}_{2}=[\mathscr{G}, \mathscr{G}]$. Let $G$ be the corresponding connected, simply connected Lie group. A family of dilations $\left\{\delta_{r}\right\}$ on $\mathscr{G}$ induces a family of algebra automorphisms, also denoted $\left\{\delta_{r}\right\}$, of $\mathscr{U}(\mathscr{G})$, the complexified universal enveloping algebra of $\mathscr{G}$. An element $P$ of $\mathscr{U}(\mathscr{G})$ is said to be homogeneous of degree $m$ with respect to $\left\{\delta_{r}\right\}$ if $\delta_{r}(P)=r^{m} P$ for all $r>0$. The set of all $P \in$ $\mathscr{U}(\mathscr{G})$ such that $P$ is homogeneous of degree $m$ with respect to a given family of dilations $\left\{\delta_{r}\right\}$ will be denoted $\mathscr{U}_{m}\left(\mathscr{G},\left\{\delta_{r}\right\}\right)$ or simply $\mathscr{U}_{m}(\mathscr{G})$ when there is no chance of confusion. We consider the elements of $\mathscr{C}(\mathscr{G})$ as left invariant differential operators on $G$.

THEOREM 2. Let $\mathscr{G}$ be a nilpotent Lie algebra of step two and $\left\{\delta_{r}\right\}$ a family of dilations on $\mathscr{G}$. If $P \in \mathscr{U}_{m}\left(\mathscr{G},\left\{\delta_{r}\right\}\right)$ is hypoelliptic, then $P^{*}$ is hypoelliptic.

When $\left\{\delta_{r}\right\}$ is the natural family of dilations for a grading $\mathscr{G}=$ $\mathscr{G}_{1} \oplus \mathscr{G}_{2}$ of $\mathscr{G}$, then this result was proved in Helffer and Nourrigat [4]. For the Heisenberg group such a result was proved in Miller [6]. It follows from this theorem that any hypoelliptic $P \in \mathscr{U}_{m}(\mathscr{G})$ is locally solvable.

The proof is based on the Helffer-Nourrigat-Rockland characterization of the hypoelliptic operators in $\mathscr{U}_{m}(\mathscr{G}): P \in \mathscr{U}_{m}(\mathscr{G})$ is hypoelliptic if and only if $\pi(P)$ is injective in $\mathscr{S}_{\pi}$ for every nontrivial irreducible unitary representation $\pi$ of $G$. (See [3] and [8]. That this result holds for arbitrary dilations is shown in [7].) We shall also need some other preliminary information before beginning the proof of Theorem 2.

By Lemma 1.2 of [7] there is a basis $\left\{X_{1}, \cdots, X_{N} ; \cdots, X_{n}\right\}$ of $\mathscr{G}$ such that each $X_{j}$ is an eigenvector for the generator $A$ of $\left\{\delta_{r}\right\}$, $\left\{X_{N+1}, \cdots, X_{n}\right\}$ spans $\mathscr{G}_{2}$, and for each $k>N$ there are $i$ and $j \leqq N$ such that $\left[X_{i}, X_{j}\right]=X_{k}$. Let $\mu_{j}$ be the eigenvalue of $A$ corresponding to $X_{j}$. If $\alpha \in N^{n}$, let $\alpha \mu=\sum \alpha_{j} \mu_{j}$ and $X^{\alpha}=X_{1}^{\alpha_{1}} \cdots X_{n}^{\alpha_{n}}$. Then $P \in \mathscr{U}_{m}(\mathscr{G})$ if and only if

$$
P=\sum_{\alpha \mu=m} a_{\alpha} X^{\alpha}
$$

for some $a_{\alpha} \in \boldsymbol{C}$.

Let $\mathscr{G}_{1}$ be the subspace of $\mathscr{G}$ spanned by $\left\{X_{1}, \cdots, X_{N}\right\}$. Letting 
$\mathscr{G}^{*}$ denote the vector space dual of $\mathscr{G}$, we define $\delta_{r}$ on $\mathscr{G}^{*}$ to be the transpose of $\delta_{r}$ on $\mathscr{G}$ for each $r>0$. Since $\mathscr{G}_{1}$ is invariant under $\left\{\delta_{r}\right\},\left\{\delta_{r}\right\}$ (on $\mathscr{G}^{*}$ ) restricts to a family of dilations on the vector space $\mathscr{G}_{1}^{*}$. For $\eta \in \mathscr{G}_{1}^{*}$ define $\|\eta\|$ as in $\S 1$. If $X \in \mathscr{G}$, let $X=X^{\prime}+X^{\prime \prime}$ where $X^{\prime} \in \mathscr{G}_{1}, X^{\prime \prime} \in \mathscr{G}_{2}$. For $\eta \in \mathscr{G}_{1}^{*}$,

$$
\pi_{\eta}(\exp X)=\exp i\left\langle\eta, X^{\prime}\right\rangle
$$

defines a unitary representation of $G$ on $C$. It follows from (2.1) that if $P \in \mathscr{U}_{m}(\mathscr{G})$, then

$$
\pi_{\delta_{r} \eta}(P)=r^{m} \pi_{\eta}(P)=\pi_{\eta}\left(\delta_{r} P\right) ; \quad \eta \in \mathscr{G}_{1}^{*} .
$$

We next recall some facts about the representation theory for $G$. More details are given in [7]. Let $\zeta \in \mathscr{G}_{2}^{*}$. Then there is a $d=d(\zeta) \leqq N / 2$ and a basis $\mathscr{B}(\zeta)=\left\{Y_{1}(\zeta), \cdots, Y_{N}(\zeta)\right\}$ for $\mathscr{G}_{1}$ such that $\mathscr{B}(\zeta)$ is orthogonal with respect to the inner product determined by the basis $\left\{X_{1}, \cdots, X_{N}\right\}$ and such that

$$
\begin{aligned}
& \left\langle\zeta,\left[Y_{j}(\zeta), Y_{j+d}(\zeta)\right]\right\rangle=1 \quad \text { for } \quad j \leqq d \\
& \left\langle\zeta,\left[Y_{j}(\zeta), Y_{k}(\zeta)\right]\right\rangle=0
\end{aligned}
$$

for all other choices $j<k \leqq N$. (In [7] we had [ $\left.Y_{j}(\zeta), Y_{j+d}(\zeta)\right]=$ $\lambda_{j}>0$. This was necessary because we wanted the basis to be orthonormal, but that is not needed here.) For any $\rho \in \boldsymbol{R}^{N-2 d}$ there is an irreducible unitary representation $\pi_{\rho, \zeta}$ of $G$ on $L^{2}\left(\boldsymbol{R}^{d}\right)$ such that

$$
\begin{array}{ll}
\pi_{\rho, \zeta}\left(Y_{j}(\zeta)\right) u(t)=\partial u / \partial t_{j}, & j \leqq d ; \\
\pi_{\rho, \zeta}\left(Y_{j+d}(\zeta)\right) u(t)=i t_{j} u(t), & j \leqq d ; \\
\pi_{\rho, \zeta}\left(Y_{j+2 d}(\zeta)\right) u(t)=i \rho_{j} u(t), & j \leqq N-2 d ; \\
\pi_{\rho, \zeta}(Z) u(t)=i\langle\zeta, Z\rangle u(t), & Z \in \mathscr{G}_{2} .
\end{array}
$$

Furthermore every irreducible unitary representation of $G$ is unitarily equivalent to $\pi_{\rho, \zeta}$ for some $\zeta \in \mathscr{G}_{2}^{*}$ and some $\rho \in \boldsymbol{R}^{N-2 d(\zeta)}$. Note that if $\zeta=0$ we obtain the representation defined by (2.2).

For $\zeta \in \mathscr{G}_{2}^{*}, t \in \boldsymbol{R}^{d}, \tau \in \boldsymbol{R}^{d}$ and $\rho \in \boldsymbol{R}^{N-2 d}, d=d(\zeta)$, let $\eta(t, \tau ; \rho, \zeta)$ be that element $\eta$ of $\mathscr{G}_{1}^{*}$ such that

$$
\begin{aligned}
& \left\langle\eta, Y_{j}(\zeta)\right\rangle=\tau_{j}, \quad\left\langle\eta, Y_{j+d}(\zeta)\right\rangle=t_{j}, \quad j \leqq d ; \\
& \left\langle\eta, Y_{j+2 d}(\zeta)\right\rangle=\rho_{j}, \quad j \leqq N-2 d .
\end{aligned}
$$

Let $f \in C^{\infty}\left(\boldsymbol{R}^{N}\right)$ satisfy $f \equiv 0$ in a neighborhood of 0 and $f \equiv 1$ outside some bounded set. Define

$$
\Phi_{\rho, \zeta}(t, \tau)=1+f(t, \tau, \rho)\|\eta(t, \tau ; \rho, \zeta)\| \text {. }
$$

Let $\zeta \in \mathscr{G}_{2}^{*}, \zeta \neq 0$, be fixed. If for all $\rho \in \boldsymbol{R}^{N-2 d}, q_{\rho} \in C^{\infty}\left(\boldsymbol{R}^{2 d}\right)$ and for all multi-indices $\alpha$ and $\beta$ there is a $C_{\alpha \beta}$ such that 


$$
\left|D_{\tau}^{\alpha} D_{t}^{\beta} q_{\rho}(t, \tau)\right| \leqq C_{\alpha \beta} \Phi_{\rho, \zeta}(t, \tau)^{k-|\alpha|}
$$

for all $(t, \tau, \rho) \in \boldsymbol{R}^{N}$ we will write " $q_{\rho} \in S_{\rho, \zeta}^{k}$ uniformly in $\rho$ ". $\mathscr{L}_{\rho, \zeta}^{k}$ is the space of pseudodifferential operators with symbols in $S_{\rho, \zeta}^{k}$; $H_{\rho, \zeta}^{k}$ the corresponding global Sobolev space as defined in [1].

It follows from (2.5), (2.6) and (2.2) that, for $X \in \mathscr{G}_{1}$,

$$
\operatorname{sym} \pi_{\rho, \zeta}(X)(t, \tau)=\pi_{\eta(t, \tau, \rho, \zeta)}(X),
$$

where sym $Q$ denotes the symbol of the operator $Q$. Let $\zeta \in \mathscr{G}_{2}^{*}$ be fixed and let $\left\{X_{1}, \cdots, X_{n}\right\}$ be the basis for $\mathscr{G}$ described at the beginning of this section. By (2.7) and (1.2),

$$
\begin{array}{ll}
\pi_{\rho, \zeta}\left(X_{j}\right) \in \mathscr{L}_{\rho, \zeta}^{\mu_{i}} & \text { uniformly in } \rho \text { if } j \leqq N, \\
\pi_{\rho, \xi}\left(X_{j}\right) \in \mathscr{L}_{\rho, \zeta}^{0} \quad \text { uniformly in } \rho \text { if } & j>N .
\end{array}
$$

Thus if $P \in \mathscr{U}_{m}(\mathscr{G})$, then $\pi_{\rho, \zeta}(P) \in \mathscr{L}_{\rho, \zeta}^{m}$ uniformly in $\rho$.

Lemma. Let $P \in \mathscr{U}_{m}(\mathscr{G})$ satisfy $\pi_{\eta}(P) \neq 0$ for each of the one dimensional unitary representations $\pi_{\eta}, \eta \in \mathscr{G}_{1}^{*}, \eta \neq 0$. Then for fixed $\zeta \in \mathscr{G}_{2}^{*}, \zeta \neq 0$, there is $a c>0$ and $a C>0$ such that

$$
\left|\operatorname{sym} \pi_{\rho, \zeta}(P)(t, \tau)\right| \geqq c \Phi_{\rho, \zeta}(t, \tau)^{m}
$$

for all $\rho \in \boldsymbol{R}^{N-2 d}$ and all $(t, \tau) \in \boldsymbol{R}^{2 d}$ such that $|t|+|\tau| \geqq C$.

Proof. Let $S=\left\{\eta \in \mathscr{G}_{1}^{*}:\|\eta\|=1\right\}$ and let $c_{1}=\min \left\{\pi_{\eta}(P): \eta \in S\right\}$. For arbitrary $\eta \in \mathscr{G}_{1}^{*}, \eta \neq 0$, let $r=\|\eta\|^{-1}$. Then $\left\|\delta_{r} \eta\right\|=1$. (2.3) implies that $\left|\pi_{\eta}(P)\right| \geqq c_{1}\|\eta\|^{m}$. Thus letting $p_{\rho, \zeta}^{\prime}(t, \tau)=\pi_{\eta(t, \tau, \rho, \zeta)}(P)$, we have

$$
\left|p_{\rho, \zeta}^{\prime}(t, \tau)\right| \geqq c_{1}\|\eta(t, \tau ; \rho, \zeta)\|^{m} .
$$

Let $p_{\rho, \zeta}=\operatorname{sym} \pi_{\rho, \zeta}(P)$. By (2.7), the pseudodifferential operator calculus, (2.9) and the remark following (2.9),

$$
p_{\rho, \zeta}-p_{\rho, \zeta}^{\prime} \in S_{\rho, \zeta}^{m-1} \quad \text { uniformly in } \rho \text {. }
$$

Now there exist $c_{2}>0$ and $C_{2}$ such that if $|t|+|\tau| \geqq C_{2}$ then $\|\eta(t, \tau ; \rho, \zeta)\|^{m} \geqq c_{2}\left(\left.\right|_{1} ^{\prime} t|+| \tau \mid\right)$ for all $\rho$. Thus, by (2.10), there exist $c_{3}>0$ and $C_{3}$ such that if $|t|+|\tau| \geqq C_{3}$, then $\left|p_{\rho, \zeta}^{\prime}(t, \tau)\right| \geqq c_{3} \Phi_{\rho, \zeta}(t, \tau)^{m}$ for all $\rho$. Also, by (2.11), it follows that given $\varepsilon>0$ there is a $C_{4}(\varepsilon)$ such that if $|t|+|\tau| \geqq C_{4}(\varepsilon)$, then for all $\rho$

$$
\left|p_{\rho, \zeta}(t, \tau)-p_{\rho, \zeta}^{\prime}(t, \tau)\right|<1 / 2 \varepsilon \Phi_{\rho, \zeta}(t, \tau)^{m} .
$$

The lemma follows by taking $C=\max \left\{C_{3}, C_{4}\left(c_{3}\right)\right\}$. 
Proof of Theorem 2. By the theorem of Helffer-NourrigatRockland, to prove $P^{*}$ hypoelliptic it suffices to show that $\operatorname{ker} \pi_{\rho, \zeta}\left(P^{*}\right)=0$ for all $\zeta \in \mathscr{G}_{2}^{*}$ and all $\rho \in \boldsymbol{R}^{N-2 d(\zeta)}$, except $\zeta=0, \rho=0$. (We consider $\pi_{\rho, \zeta}(P)$ and $\pi_{\rho, \zeta}\left(P^{*}\right)$ as bounded operators from $H_{\rho, \zeta}^{m}$ to $\left.H_{\rho, \zeta}^{0}\right)$. If $\zeta=0$, then

$$
\pi_{\rho, \zeta}\left(P^{*}\right)=\overline{\pi_{\rho, \zeta}(P)} \neq 0
$$

for all $\rho \neq 0$. If $\zeta \neq 0$, then by Theorem 7.2 of [1] and the above lemma, $\pi_{\rho, \zeta}(P)$ is Fredholm for all $\rho$. Also by Remark 1.4 of [4] and the Helffer-Nourrigat-Rockland Theorem, $\operatorname{ker} \pi_{\rho, \zeta}(P)=\operatorname{ker} \pi_{\rho, \zeta}(P) \cap$ $\mathscr{S}_{\pi}=0$. Hence it suffices to prove that ind $\pi_{\rho, \zeta}(P)=0$.

We consider first the case when $d=d(\zeta)<N / 2$. Let $q_{\rho, \zeta}=$ $\operatorname{sym} \pi_{\rho, \zeta}\left(P^{*}\right)$. By (2.12) and the above lemma there is a $c>0$ and a $C$ such that $\left|q_{\rho, \xi}(t, \tau)\right| \geqq c \Phi_{\rho, \zeta}(t, \tau)^{m}$ for all $(t, \tau, \rho) \in \boldsymbol{R}^{N}$ with $|t|+|\tau| \geqq C$. Choose $f \in C^{\infty}\left(\boldsymbol{R}^{2 d}\right)$ such that $f(t, \tau) \equiv 0$ if $|t|+|\tau| \leqq C$, $f(t, \tau) \equiv 1$ if $|t|+|\tau| \geqq 2 C$. Let $a_{\rho, \zeta}=f q_{\rho, \zeta}^{-1}$. Then $a_{\rho, \zeta} \in S_{\rho, \zeta}^{-m}$ uniformly in $\rho$ and $b_{\rho, \zeta}=1-a_{\rho, \zeta} \circ q_{\rho, \zeta} \in S_{\rho, \zeta}^{-1}$ uniformly in $\rho$, where $p \circ q$ denotes the symbol of $p(t, D) q(t, D)$. Let $\psi(\tau)=\left(1+|\tau|^{2}\right)^{1 / 2 m}$. There is a $C>0$ (depending on $\zeta$ ), such that $\psi(\tau) \leqq C \Phi_{\rho, \zeta}(t, \tau)$ and, by (2.8), such that $|\rho|^{\varepsilon} \leqq C \Phi_{\rho, \zeta}(t, \tau)$ for all $(t, \tau, \rho) \in \boldsymbol{R}^{N}$, where $\varepsilon=\min \left\{1 / \mu_{j}\right.$ : $i \leqq j \leqq N\}$. Thus $a_{\rho, \zeta} \in S_{\psi}^{0}$ uniformly in $\rho$ and $|\rho|^{8} b_{\rho, \zeta} \in S_{\psi}^{0}$ uniformly in $\rho$. By the $L^{2}$ boundedness theorem for pseudodifferential operators there is a $C_{1}$ such that $\left\|a_{\rho, 5}(t, D) u\right\| \leqq C_{1}\|u\|$ and $|\rho|^{k}\left\|b_{\rho, 5}(t, D) u\right\| \leqq$ $C_{1}\|u\|$, for all $u \in L^{2}\left(\boldsymbol{R}^{d}\right)$ and all $\rho$. Thus if $|\rho|^{\varepsilon} \geqq 2 C_{1}$,

$$
\begin{aligned}
\|u\| & \leqq\left\|a_{\rho, \zeta}(t, D) \pi_{\rho, \zeta}\left(P^{*}\right) u\right\|+\left\|b_{\rho, \zeta}(t, D) u\right\| \\
& \leqq C_{1}\left\|\pi_{\rho, \zeta}\left(P^{*}\right) u\right\|+1 / 2\|u\| .
\end{aligned}
$$

Hence $\pi_{\rho, \zeta}\left(P^{*}\right)$ is injective and thus ind $\pi_{\rho, 5}(P)=0$ if $|\rho|^{\varepsilon} \geqq 2 C_{1}$. Since ind $\pi_{\rho, \zeta}(P)$ is independent of $\rho$, ind $\pi_{\rho, \zeta}(P)=0$ for all $\rho \in R^{N-2 d}$.

If $d=d(\zeta)=N / 2$, we write $\pi_{\zeta}$ for $\pi_{0, \zeta}$. Define $\varphi: \boldsymbol{R}_{t}^{d} \oplus \boldsymbol{R}_{\tau}^{d} \rightarrow \mathscr{G}_{1}^{*}$ by $\varphi(t, \tau)=\eta(t, \tau ; 0, \zeta)$, as defined before (2.6). Let $\delta_{r}^{\prime}=\varphi^{-1} \circ \delta_{r} \circ \varphi$. Then $\left\{\delta_{r}^{\prime}\right\}$ is a family of dilations on $\boldsymbol{R}^{2 d}$. Let $p_{5}^{\prime}(t, \tau)=\pi_{\eta(t, \tau ; 0,5)}(P)$. It follows from (2.3) that $p_{\zeta}^{\prime}$ is homogeneous of degree $m$ with respect to $\left\{\delta_{r}^{\prime}\right\}$ and by $(2.12) p_{\zeta}^{\prime}$ is $\Phi_{\zeta}$-elliptic. Since $p_{\zeta}^{\prime}-\operatorname{sym} \pi_{\zeta}(P) \in$ $S_{\zeta}^{m-1}$ we can apply Theorem 1 to find ind $\pi_{\zeta}(P)$. If $d>1$, then ind $\pi_{\zeta}(P)=0$.

If $d=1$ and $\mathscr{B}(\zeta)=\left\{Y_{1}(\zeta), Y_{2}(\zeta)\right\}$, set $Y_{1}(-\zeta)=Y_{2}(\zeta), Y_{2}(-\zeta)=$ $Y_{1}(\zeta)$. Then $\mathscr{B}(-\zeta)=\left\{Y_{1}(-\zeta), Y_{2}(-\zeta)\right\}$ satisfies $(2.4)$ for $-\zeta$. Also $\eta(t, \tau ;-\zeta)=\eta(\tau, t ; \zeta)$ and $p_{-\zeta}^{\prime}(t, \tau)=p_{\zeta}^{\prime}(\tau, t)$. By Theorem 1

$$
2 \pi \text { ind } \pi_{-\zeta}(P)=\Delta_{\Gamma} \arg p_{-\zeta}^{\prime}=-\Delta_{\Gamma} \arg p_{\zeta}^{\prime}=-2 \pi \text { ind } \pi_{\zeta}(P) \text {. }
$$

But ker $\pi_{\zeta}(P)=\operatorname{ker} \pi_{-\zeta}(P)=0$ implies ind $\pi_{\zeta}(P) \geqq 0$ and ind $\pi_{-\zeta}(P) \geqq 0$. Thus ind $\pi_{\zeta}(P)=0$. 


\section{REFERENCES}

1. R. Beals, A general calculus of pseudodifferential operators, Duke Math. J., 42 (1975), 1-42.

2. V. Grushin, On a class of hypoelliptic pseudodifferential operators degenerate on a submanifold, Mat. Sb,, 84 (1971), 111-134; Math. USSR-Sb., 13 (1971), 155-185.

3. B. Helffer and J. Nourrigat, Caractérization des opérateurs hypoelliptiques homogènes invariants à gauche sur un groupe de Lie nilpotent gradué, Comm. P.D.E., 4 (1979), 899-958.

4. - Théorèmes d'indice pour des opérateurs différentiels a coefficients polynomiaux, (preprint).

5. L. Hormander, The Weyl calculus of pseudo-differential operators, Comm. Pure Appl. Math., 32 (1979), 359-443.

6. K. Miller, Hypoellipticity on the Heisenberg group, J. Functional Analysis, 31 (1979), 306-320.

7. - Parametrices for hypoelliptic operators on step two nilpotent Lie groups, Comm. P.D.E., 5 (1980), 1153-1184.

8. C. Rockland, Hypoellipticity on the Heisenberg group: representation-theoretic criteria, Trans. Amer. Math. Soc., 240 (1978), 1-52.

Received November 20, 1980.

Wichita State UNIVERSity

WICHITA, KS 67208 


\title{
PACIFIC JOURNAL OF MATHEMATICS
}

\section{EDITORS}

\author{
DONALD BABBITT (Managing Editor) \\ University of California \\ Los Angeles, CA 90024 \\ Hugo RossI \\ University of Utah \\ Salt Lake City, UT 84112 \\ C. C. MOORE and Arthur Agus \\ University of California \\ Berkeley, CA 94720
}

\section{J. DugundjI}

Department of Mathematics

University of Southern California

Los Angeles, CA 90007

R. FinN and J. Milgram

Stanford University

Stanford, CA 94305

\section{ASSOCIATE EDITORS}
R. ARENS
E. F. BeCKENBACH
B. H. NeumanN
F. WOLF
K. YoshidA

\section{SUPPORTING INSTITUTIONS}

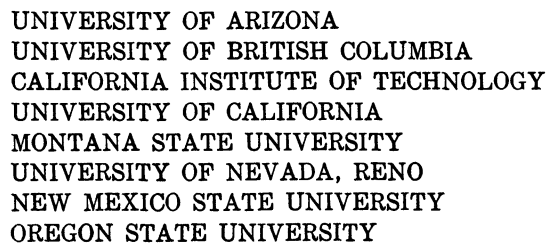

UNIVERSITY OF ARIZONA

UNIVERSITY OF BRITISH COLUMBIA

CALIFORNIA INSTITUTE OF TECHNOLOGY

UNIVERSITY OF CALIFORNIA

MONTANA STATE UNIVERSITY

UNIVERSITY OF NEVADA, RENO

NEW MEXICO STATE UNIVERSITY

OREGON STATE UNIVERSITY

\author{
UNIVERSITY OF OREGON \\ UNIVERSITY OF SOUTHERN CALIFORNIA \\ STANFORD UNIVERSITY \\ UNIVERSITY OF HAWAII \\ UNIVERSITY OF TOKYO \\ UNIVERSITY OF UTAH \\ WASHINGTON STATE UNIVERSITY \\ UNIVERSITY OF WASHINGTON
}

The Supporting Institutions listed above contribute to the cost of publication of this Journal, but they are not owners or publishers and have no responsibility for its content or policies.

Mathematical papers intended for publication in the Pacific Journal of Mathematics should be in typed form or offset-reproduced, (not dittoed), double spaced with large margins. Please do not use built up fractions in the text of the manuscript. However, you may use them in the displayed equations. Underline Greek letters in red, German in green, and script in blue. The first paragraph or two must be capable of being used separately as a synopsis of the entire paper. Please propose a heading for the odd numbered pages of less than 35 characters. Manuscripts, in triplicate, may be sent to any one of the editors. Please classify according to the scheme of Math. Reviews, Index to Vol. 39. Supply name and address of author to whom proofs should be sent. All other communications should be addressed to the managing editor, or Elaine Barth, University of California, Los Angeles, California, 90024.

50 reprints to each author are provided free for each article, only if page charges have been substantially paid. Additional copies may be obtained at cost in multiples of 50 .

The Pacific Journal of Mathematics is issued monthly as of January 1966. Regular subscription rate: $\$ 102.00$ a year (6 Vols., 12 issues). Special rate: $\$ 51.00$ a year to individual members of supporting institutions.

Subscriptions, orders for numbers issued in the last three calendar years, and changes of address shoud be sent to Pacific Journal of Mathematics, P.O. Box 969, Carmel Valley, CA 93924, U.S.A. Old back numbers obtainable from Kraus Periodicals Co., Route 100, Millwood, NY 10546.

\section{PUBLISHED BY PACIFIC JOURNAL OF MATHEMATICS, A NON-PROFIT CORPORATION}

Printed at Kokusai Bunken Insatsusha (International Academic Printing Co., Ltd.). 8-8, 3-chome, Takadanobaba, Shinjuku-ku, Tokyo 160, Japan. 


\section{Pacific Journal of Mathematics}

Vol. 99, No. $2 \quad$ June, 1982

Thomas E. Armstrong and Karel Libor Prikry, On the semimetric on a

Boolean algebra induced by a finitely additive probability measure . . . .249

Walter Russell Bloom, Strict local inclusion results between spaces of

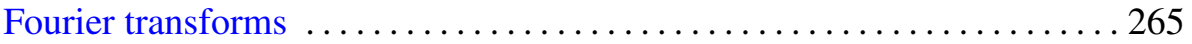

Richard Clark Brown, Notes on generalized boundary value problems in

Banach spaces. II. Infinite-dimensional extension theory ........... 271

Sui Sun Cheng, Isoperimetric eigenvalue problem of even order differential

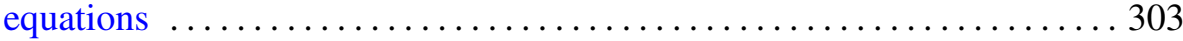

Lung O. Chung and Jiang Luh, Derivations of higher order and

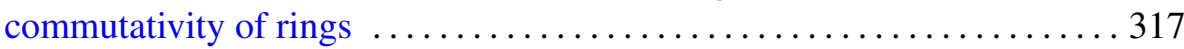

Ali Ahmad Fora, A fixed point theorem for product spaces . . . . . . . . . 327

Barry J. Gardner, Radical classes of regular rings with Artinian primitive

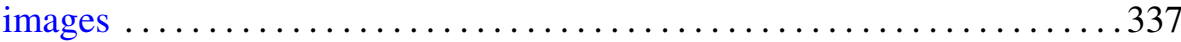

John Brady Garnett and Peter Wilcox Jones, BMO from dyadic BMO . . . 351

Allen E. Hatcher, On the boundary curves of incompressible surfaces . . . . 373

Richard Howard Hudson and Kenneth S. Williams, Resolution of

ambiguities in the evaluation of cubic and quartic Jacobsthal sums .....379

Viktor Losert, Counter-examples to some conjectures about doubly

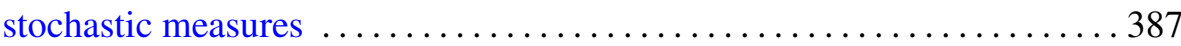

Kenneth Derwood Magill, Jr., P. R. Misra and Udai Bhan Tewari,

Structure spaces for sandwich semigroups

Mark Mandelker, Continuity of monotone functions

Kenneth Guy Miller, An index theorem and hypoellipticity on nilpotent Lie groups ......................................... 419

Evelyn M. Nelson, Homomorphisms of mono-unary algebras . . . . . . . . . 427

Marvin E. Ortel, The support of an extremal dilatation . . .

R. S. Pathak and O. P. Singh, Finite Hankel transforms of distributions . . . 439

Richard Cole Penney, The theory of ad-associative Lie algebras

Linda Ruth Sons, Zero distribution of functions with slow or moderate

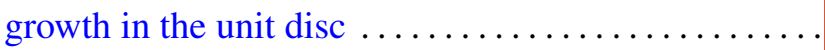

Russell Bruce Walker, Transversals to laminations 